\title{
Unusual case of a giant serous oligocystic adenoma with communication to main pancreatic duct
}

\author{
Ruquaya Mir, Vikram Pratap Singh
}

\begin{abstract}
Benign serous cystic tumors of the pancreas are rare and include microcystic serous adenoma, serous oligocystic adenoma (SOA), illdemarcated adenoma, and macrocystic serous cystadenoma. Microcystic serous adenoma usually present are either unilocular cyst or single lesion containing fewer cysts of more than $2 \mathrm{~cm}$ in diameter. It is a rare tumor which usually varies from $2-10 \mathrm{~cm}$ in size and at times difficult to differentiate from mucinous cyst. With firm diagnosis small asymptomatic SOAs could be managed without surgery. We describe here a case of middle aged male with a giant serous oligocystic adenoma of $15 \mathrm{~cm}$ size arising from head of pancreas producing pressure symptoms. Endoscopic ultrasound and cyst fluid tumor markers were suggestive of serous cystadenoma, computed tomography (CT) and magnetic resonance cholangiopancreatography (MRCP) scan were indicative of mucinous adenoma showing unilocular cyst with dilated common bile duct (CBD), main pancreatic duct (MPD) and communication of cyst with main pancreatic duct. A pancreaticoduodenectomy was done. Histopathology reported the lesion as serous oligocystic adenoma. We are presenting this case due to unusual features, a large unilocular cyst of more than $15 \mathrm{~cm}$ in size presented with waxing/ waning of surgical obstructive jaundice and on
\end{abstract}

Ruquaya Mir $^{1}$, Vikram Pratap Singh ${ }^{1}$

Affiliations: ${ }^{1}$ Senior Consultant Oncology, Indraprastha Apollo Hospitals, New Delhi, India.

Corresponding Author: Ruquaya Mir, Senior Consultant Oncology, Indraprastha Apollo Hospitals, New Delhi, India. Email: ruquaya.mir@hotmail.com

Received: 19 August 2014

Accepted: 04 September 2014

Published: 21 November 2014
MRCP there was a communication of cyst with MPD with cyst wall thickness of $1 \mathrm{~cm}$ (approx.).

Keywords: Common bile duct (CBD), Giant serous oligocystic adenoma, Jaundice, Magnetic resonance cholangiopancreatography (MRCP), Main pancreatic duct (MPD), Ttreatment

\section{How to cite this article}

Mir R, Singh VP. Unusual case of a giant serous oligocystic adenoma with communication to main pancreatic duct. Int $\mathrm{J}$ Hepatobiliary Pancreat Dis 2014;4:57-61.

Article ID: 100023IJHPDRM2014

$* * * * * * * * *$

doi:10.5348/ijhpd-2014-23-CR-11

\section{INTRODUCTION}

Cystic neoplasm of pancreas is relatively a common entity and is second only to adenocarcinoma of pancreas. The major histological subtypes are serous cystic neoplasm (SCN), intraductal papillary mucinous neoplasm (IPMNs) and mucinous cystic neoplasm (MCNs) [1]. Rare entities include solid pseudopapillary neoplasm, cystic pancreatic endocrine neoplasm, cystic ductal adenocarcinoma and acinar cell cystadenoma. All cystic neoplasm are considered malignant or premalignant except SCNs which are almost invariably benign [2]. Preoperative diagnosis of the cyst type is necessary to determine the line of treatment. Serous cystic tumors of the pancreas could be microcystic serous adenoma and macrocystic or oligocystic serous cystadenoma. Lewandrowski first described the macrocystic or oligocystic variants of serous cystadenoma which by 
definition consist of unilocular cyst or a single lesion containing $<6$ individual cysts, each of more than $2 \mathrm{~cm}$ in diameter $[3,4]$. The more frequent microcystic variants contains at least six cysts each measuring less than $2 \mathrm{~cm}$ in diameter with sponge like honeycomb appearance or central scar or both. Surgical treatment should not be considered unless tumor is symptomatic or the benefit of the operation outweighs risk because of the invariably benign nature of serous oligocystic adenomas [5]. But the establishment of diagnosis of serous oligocystic adenomas (SOAs) is sometimes difficult despite the availability of newer diagnostic methods. Here presenting a case of symptomatic giant cystic neoplasm of pancreatic head with communication to main pancreatic duct which has been operated with a suspected diagnosis of mucinous cyst adenoma, but histopathology reported it as a serous oligocystic adenoma.

\section{CASE REPORT}

A 41-year-old male patient with borderline hypertension, presented to us with complaints of jaundice and postprandial vomiting started one and a half months before without any fever or alteration of bowel, bladder habit. But he noticed a spontaneous partial relief of his symptoms for 10-15 days before he presented to us. Though he has some loss of appetite and weight, but his performance status was otherwise good. At the time of admission patient was icteric with serum bilirubin of $3.5 \mathrm{mg} / \mathrm{dL}$, alkaline phosphate 1414 U/1 and GGTP 474 U/l. Physical examination revealed a large firm mass present in upper abdomen extending from right hypochondrium, epigastrium to right lumber and umbilical region. It was non-tender, partially fixed, and dull on percussion without any abnormal pulsation. Contrast computed tomography (CT) scan revealed a well-defined unilocular cyst measuring 15.2x12.1x11.6 $\mathrm{cm}$ arising from the head of pancreas pressing over the duodenum and common bile duct (CBD) with dilatation of main pancreatic duct (MPD) (Figure 1). Magnetic resonance cholangiopancreatography (MRCP) revealed dilated CBD and MPD with communication between the cyst and MPD (Figure 2). Endoscopic ultrasound showed unilocular cyst without further specification. Endoscopic ultrasound guided fine-needle aspiration cytology (FNAC) was done for fluid analyses, biochemistry and cytology. Cytology of cyst fluid did not show any malignant cell, cyst fluid CEA was $1.4 \mathrm{ng} / \mathrm{mL}$. Fluid amylase (6o IU/l) and CA19-9 (14.71 U/mL) were within normal limits. In view of obstructive symptoms due to giant size of the lesion pancreaticoduodenectomy was planned. On exploration a huge unilocular cyst measuring 15 $\mathrm{cm}$ in greatest dimension was found, displacing the duodenum and pylorus, superior mesenteric vessels and portal vein. Cyst was densely adhered to anterior surface of right kidney, inferior vena cava (IVC) and portal vein. Pancreaticoduodenectomy was performed
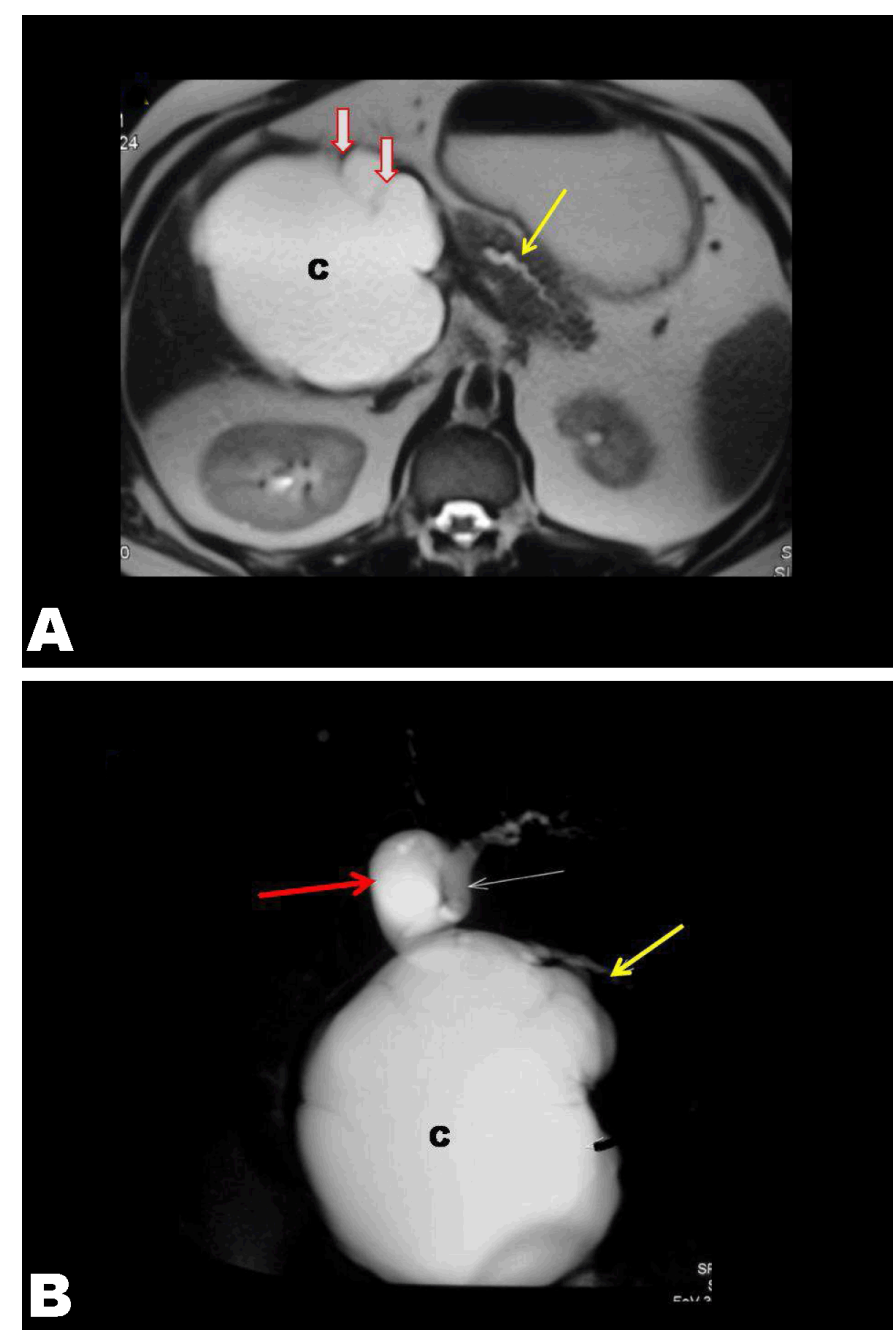

Figure 1: (A) Axial T2-weighted (B) Coronal magnetic resonance cholangiopancreatography images reveal a sharply defined T2 hyper-intense cystic lesion in 'c' pancreatic head with internal septations (red open arrows) causing common bile duct (CBD) (white arrow) and pancreatic duct (yellow arrow) dilatation. Red arrow indicates gallbladder.

with Roux-en-Y reconstruction. Postoperative recovery was uneventful and the patient was discharged on sixth postoperative day. Biopsy revealed a solitary cyst filled with hemorrhagic fluid with maximum wall thickness of $1 \mathrm{~cm}$. Microscopic examination showed a cuboidal epithelial lining of cyst wall with focal papillary formation and multi-layering. Cells showed moderate clear cytoplasm and uniform round nuclei without any pleomorphism or mitosis. Cyst wall showed dense fibrosis and patchy mild chronic inflammatory cell infiltrate. Final histopathological diagnosis was serous oligocystic adenoma head of pancreas.

\section{DISCUSSION}

Since the first description of serous and mucinous cystadenoma by Compagno and Oertel, many tried to characterize these variants on radiological, pathological and immunohistochemical basis $[5,6]$. But many a 

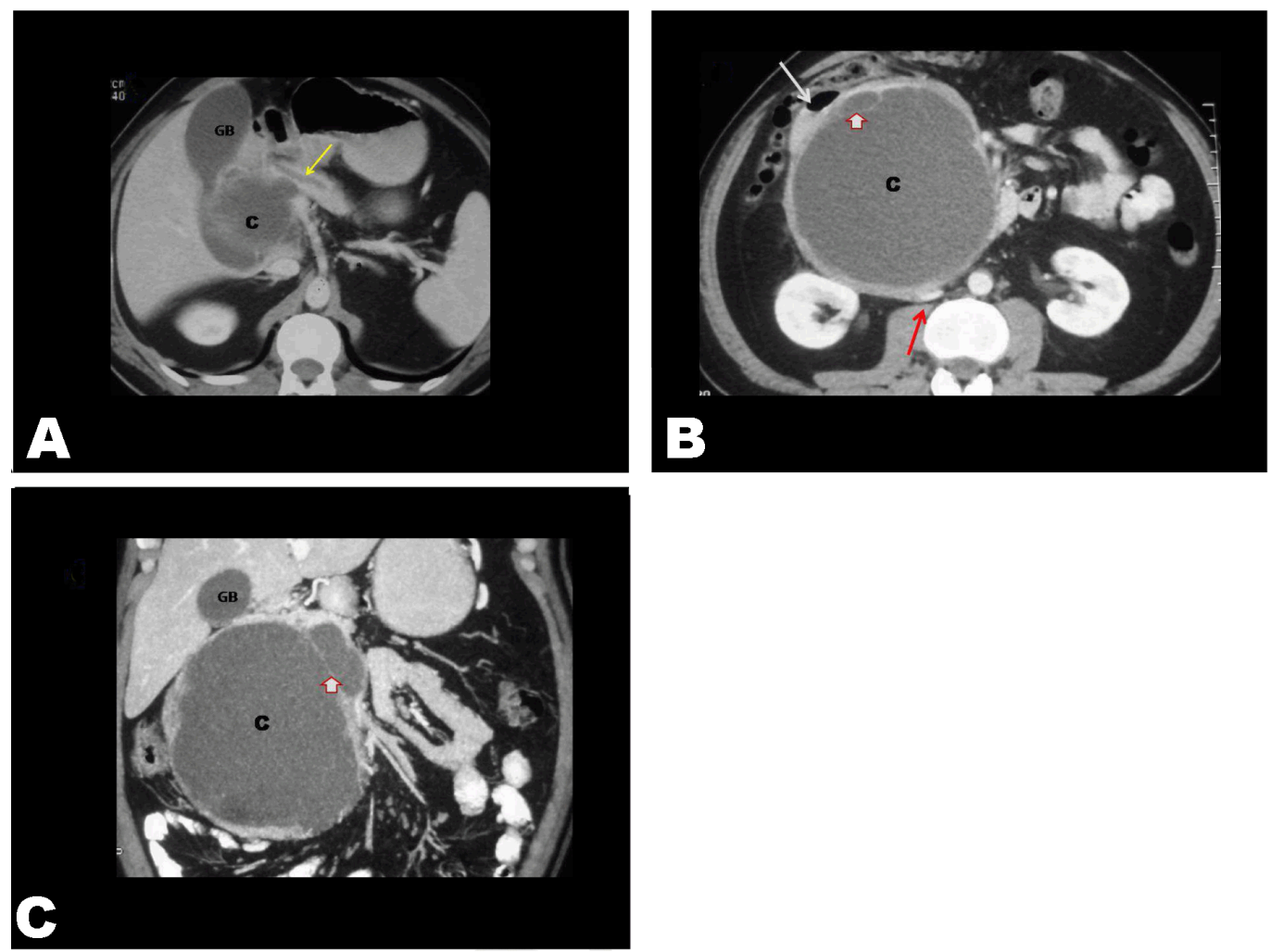

Figure 2: (A, B) Contrast-enhanced axial, and coronal (C) Computed tomography (CT) image in a porto-venous phase revealing a well-marginated, rounded hypodense cystic lesion with lobulated margins in the pancreatic head ' $\mathrm{C}$ ', showing few enhancing internal septations (red open arrows). Lesion displaces the duodenum (white arrow) antero-superiorly and flattens the anterior wall of inferior vena cava (red arrow). Main pancreatic duct (yellow arrow) is dilated in the pancreatic body.

times diagnosis remains elusive even with advanced measures until histopathology, as in the present case. Serous oligocystic adenoma is a rare entity and comprise $10-30 \%$ of serous cyst adenomas [7]. Half of these cases are asymptomatic and diagnosed incidentally. Though microcystic variant may show radiographically visible multiple cysts with or without central scarring, SOAs have few characteristic features. However, distinction is crucial as small asymptomatic SOAs can be managed conservatively. Typical SOAs on imaging appears as a small unilocular or bilocular cyst with a thin wall $(2 \mathrm{~mm})$ which lacks mural nodule or calcification [8]. SOAs can be mistaken for mucinous cystic neoplasm, pseudocyst or intraductal papillary mucinous tumor because of relatively large cystic spaces [9]. Giant serous cystadenoma refer to a cystic pancreatic tumor with a diameter of $10 \mathrm{~cm}$ or more, which is very rare in comparison to mean tumor diameter of 4-5 cm [10]. They may produce symptom due to pressure affect to surrounding structures. Communication to main pancreatic duct (MPD) is one of the characteristic features of IPMNs and rarely seen in case of MCN and SCN. But it is a very unusual in case of serous cystadenoma and has been reported only in $0.6 \%$ cases in endoscopic retrograde cholangiopancrteatography (ERCP) in a series of 144 cases [11]. Waxing and Waning of jaundice may be because of decompression of cyst in MPD. The CT and MRI features of serous oligocystic tumors are documented and diagnosis of cystic neoplasm in most of cases are done on the basis of imaging. Kim et al. [12] and Cohen-Scali et al. [13] described the characteristics CT findings which could differentiate SOAs and MCNs, namely location at head, a lobulated contour and absence of wall enhancement. Some authors regarded MRI as a better technique as it can demonstrate septa within a lesion with greater accuracy and could better evaluate the tissue characteristic like mucinous and serous fluid, solid component and other aspects [10, $11,14,15]$. Several authors regarded cyst wall thickness as an important differentiating characteristic of serous and MCNs and observed that SOAs have very thin cyst wall (2-4 $\mathrm{mm})$ as compared to MCNs or pseudocyst [8, 11]. In our case, cyst wall was thickened (approx. $10 \mathrm{~mm}$ ) with intense fibrotic reaction which is unusual of SOAs. Though transabdominal ultrasound could be of some use in large cyst to delineate multicystic characteristic or mural nodules, but most of the time it is of minimum use either due to smaller cyst size or due to deeper location and overlying bowel loops. Rather endoscopic ultrasound is considered as an ideal investigation as it could provide high resolution morphologic imaging and guide for FNAC [9]. ERCP though not required in every case, but may be helpful to differentiate IPMN which demonstrate patulous ampulla pouring out mucin with diffusely dilated pancreatic ducts.

A study revealed cyst fluid carcinoembryonic antigen (CEA) of more than $192 \mathrm{ng} / \mathrm{mL}$ had sensitivity, specificity and diagnostic accuracy of $73 \%, 84 \%$ and $79 \%$, respectively for mucinous cyst [16]. A value less than 5 
$\mathrm{ng} / \mathrm{mL}$ have been described as highly specific for nonmucinous cyst [16]. In addition, cyst fluid mucin and cytology may provide valuable information to distinguish serous from mucinous cyst.

On histopathological examination SOAs appear grossly as a cystic mass of 4-10 cm, usually unilocular, with cut surface showing one or few macroscopically visible cysts of variable sizes usually more than $2 \mathrm{~cm}$, filled with watery or brownish fluid. The irregularly arranged cysts are separated by broad septa of fibrous stroma that lacks a central stellate scar. Microscopically, serous oligocystic adenoma shows the cystic spaces lined by single layer of cuboidal flattened epithelial cells with clear cytoplasm and rarely eosinophilic. The nuclei are centrally located, round to oval in shape, uniform, and have inconspicuous nucleoli The cells contain abundant intracytoplasmic glycogen which can be demonstrated by periodic acid Schiff stain without diastase digestion. The stromal frame work is well developed and often hyalinized $[7,17]$.

Treatment is dependent on the symptoms, results of radiologic and cyst fluid analysis. Grossly, surgery is advisable in suspected MCNs, IPMNs, cyst with mass, hemorrhage or mural nodule, duct obstruction or cyst rim calcification [18]. Though asymptomatic serous cystadenoma with classic features can safely be followed, but some authors nevertheless advised surgery for a serous cyst adenoma more than $4 \mathrm{~cm}$ diameter because of higher median growth rate [19]. In asymptomatic lesion, diagnosis with certainty is of utmost importance and role of complex diagnostic procedures like endoscopic ultrasound and fine-needle aspiration in this kind of situations is worth doing to defer a major pancreatic surgery. However, situation where scenario remains cloudy despite of all measures, exploration is indicated even if serous cyst adenoma is discovered [20].

\section{CONCLUSION}

Serous oligocystic adenomas are rare variant of cystic serous neoplasm of pancreas which, though itself is a low risk tumor, but at times has many overlapping features of high risk minimal change nephrotic syndrome (MCNs) or intraductal papillary mucinous neoplasms (IPMNs) as seen in the presenting case. Though surgery becomes unavoidable in certain situation due to the size of the tumor or symptoms caused by it, firm preoperative diagnosis nevertheless helps to decide the extent of surgery more confidently. Careful approach to patient with diligent use of investigation could lessen the confusion and apprehension among patients as well as in surgeon.

$* * * * * * * * *$

\section{Author Contributions}

Ruquaya Mir - Substantial contributions to conception and design, Acquisition of data, Analysis and interpretation of data, Drafting the article, Revising it critically for important intellectual content, Final approval of the version to be published

Vikram Pratap Singh - Acquisition of data, Drafting the article, Revising it critically for important intellectual content, Final approval of the version to be published

\section{Guarantor}

The corresponding author is the guarantor of submission.

\section{Conflict of Interest}

Authors declare no conflict of interest.

\section{Copyright}

(C) 2014 Ruquaya Mir. This article is distributed under the terms of Creative Commons Attribution License which permits unrestricted use, distribution and reproduction in any medium provided the original author(s) and original publisher are properly credited. Please see the copyright policy on the journal website for more information.

\section{REFERENCES}

1. Brugge WR, Lauwers GY, Sahani D, Fernandezdel Castillo C, Warshaw AL. Cystic neoplasm of the pancreas. N Engl J Med 2004;351(12):1218-26.

2. Pyke CM, van Heerden JA, Colby TV, Sarr MG, Weaver AL. The spectrum of serous cystadenoma of the pancreas: Clinical, pathological, and surgical aspects. Ann Surg 1992 Feb;215(2):132-9.

3. Lewandrowski K, Warshaw A, Compton C. Macrocystic serous cystadenoma of pancreas: A morphologic variant differing from microcystic adenoma. Hum Pathol 1992 Aug;23(8):871-5.

4. Huh JR, Chi JG, Jung KC, Choe KJ, Yoon YB. Macrocystic serous cystadenoma of pancreas--a case report. J Korean Med Sci 1994 Feb;9(1):78-5.

5. Yamaguchi H, Ishigami K, Inoue T, et al. Three Cases of Serous Oligocystic Adenomas of the Pancreas; Evaluation of Cyst Wall Thickness for Preoperative Differentiation from Mucinous Cystic Neoplasms. J Gastrointest Cancer 2007;38(1):52-8.

6. Compagno J, Oertel JE. Microcystic adenomas of pancreas (Glycogen rich cystadenomas): A clinicopathological study of 34 cases. Am J Clin Pathol 1978 Mar;69(3):289-98.

7. Goh BK, Tan YM, Yap WM, et al. Pancreatic serous oligocystic adenomas: Clinicopathologic features and a comparison with serous microcystic adenomas and mucinous cystic neoplasms. World J Surg 2006 Aug;30(8):1553-9.

8. Brugge WR, Lewandrowski K, Lee-Lewandrowski E, et al. Diagnosis of pancreatic cystic neoplasms: A report of the cooperative pancreatic cyst study. Gastroenterology 2004 May;126(5):1330-6.

9. O’Toole D, Palazzo L, Hammel P, et al. Macrocystic pancreatic cystadenoma: The role of EUS and cyst fluid analysis in distinguishing mucinous and serous lesions. Gastrointest Endosc 2004 Jun;59(7):823-9.

10. Schulz HU, Kellner U, Kahl S, et al. A giant pancreatic serous microcystic adenoma with 20 years follow-up. Langenbecks Arch Surg 2007 Mar;392(2):209-13. 
11. Berman L, Mitchell KA, Israel G, Salem RR. Serous cystadenoma in communication with the pancreatic duct: an unusual radiologic and pathologic entity. J Clin Gastroenterol $2010 \mathrm{Jul}$;44(6):e133-5.

12. Kim SY, Lee JM, Kim SH, et al. Macrocystic neoplasms of the pancreas: CT differentiation of serous oligocystic adenoma from mucinous cystadenoma and intraductal papillary mucinous tumor. AJR Am J Roentgenol 2006 Nov;187(5):1192-8.

13. Cohen-Scali F, Vilgrain V, Brancatelli G, et al. Discrimination of unilocular macrocystic serous cystadenoma from pancreatic pseudocyst and mucinous cystadenoma with CT: Initial observations. Radiology 2003 Sep;228(3):727-33.

14. Kim YH, Saini S, Sahani D, Hahn PF, Mueller PR, Auh YH. Imaging diagnosis of cystic pancreatic lesions: Pseudocyst versus nonpseudocyst. Radiographics 2005 May-Jun;25(3):671-85.

15. Choi JY, Kim MJ, Lee JY, et al. Typical and atypical manifestations of serous cystadenoma of the pancreas: Imaging findings with pathologic correlation. AJR
Am J Roentgenol 2009 Jul;193(1):136-42.

16. Van Der Waaij LA, Van Dullemen HM, Porte RJ. Cyst fluid analysis in the differential diagnosis of pancreatic cystic lesions: A pooled analysis. Gastrointest Endosc 2005 Sep;62(3):383-9.

17. Khadaroo R, Knetman N, Joy S, Nguyen GK. Macrocystic serous adenoma of the pancreas. Pathol Res Pract 2002;198(7):485-8.

18. Walsh RM, Vogt DP, HendersonJM, etal. Management of suspected pancreatic cystic neoplasms based on cyst size. Surgery 2008 Oct;144(4):677-84.

19. Tseng JF, Warshaw AL, Sahani DV, Lauwers GY, Rattner DW, Fernandez-Del Castillo C. Serous cystadenoma of the pancreas: Tumor growth rates and recommendations for treatment.Ann Surg 2005 Sep;242(3):413-9.

20. Galanis C, Zamani A, Cameron JL, et al. Resected serous cystic neoplasms of the pancreas: A review of 158 patients with recommendations for treatment. J Gastrointest Surg 2007 Jul;11(7):820-6.

\section{ABOUT THE AUTHORS}

Article citation: Mir R, Singh VP. Unusual case of a giant serous oligocystic adenoma with communication to main pancreatic duct. Int J Hepatobiliary Pancreat Dis 2014;4:57-61.

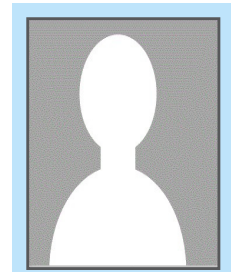

Ruquaya Mir is Senior Consultant Surgical Oncology Indraprastha Apollo Hospitals, New Delhi, India. She earned the undergraduate degree MBBS, DNB from Kashmir University, J\&K, India and postgraduate degree Diplomate National Board from National Board Examination, New Delhi India. She has published 10 research papers in national and international academic journals and authored books. Her research interests include Pancreatic Cancer, Oesophageal cancer, Head \& Neck Cancer. She intends to pursue in future. Email: ruquaya.mir@hotmail.com

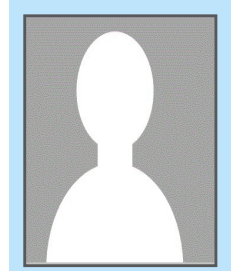

Vikram Pratap Singh is Senior Consultant Surgical Oncology at Indraprastha Apollo Hospitals, New Delhi India. He earned the undergraduate degree MBBS, MS, FRCS from University of Lucknow, UP, India and postgraduate degree from King George Medical College, University of Lucknow, UP, India, FRCS from Glasgow. He has published 32 research papers in national and international academic journals and authored books. His research interests include Pancreatic Cancer, Head \& Neck Cancer, Oesophageal Cancer. He intends to pursue in future. Email: vikrampsingh@hotmail.com, drvikrampsingh@gmail.com

Access full text article on other devices

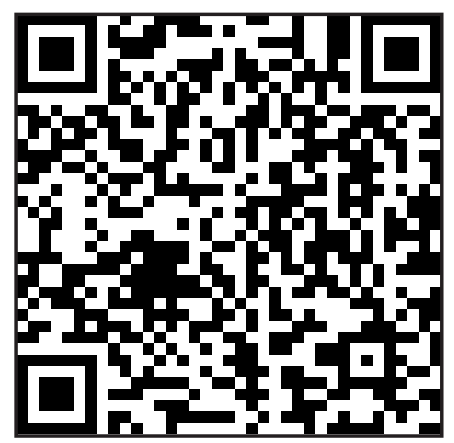

Access PDF of article on other devices

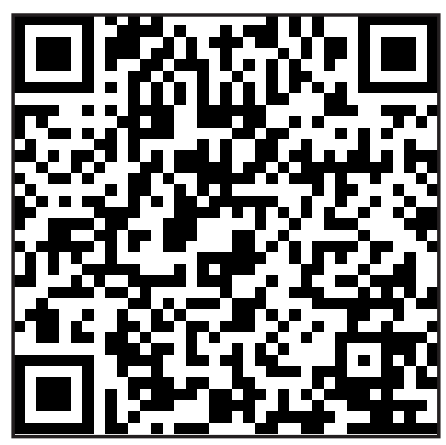

